\title{
Micro operators to boost local service delivery in $5 G$
}

\author{
Marja Matinmikko ${ }^{1}$, Matti Latva-aho ${ }^{1}$, Petri Ahokangas ${ }^{2}$, Seppo Yrjölä3, Timo Koivumäki ${ }^{2}$ \\ ${ }^{1}$ Centre for Wireless Communications (CWC), University of Oulu, Oulu, Finland \\ ${ }^{2}$ Oulu Business School, Martti Ahtisaari Institute (MAI), Oulu, Finland \\ ${ }^{3}$ Nokia, Oulu, Finland \\ Corresponding author: Marja Matinmikko, marja.matinmikko@oulu.fi, +358445136678
}

\begin{abstract}
Future digital society depends heavily on timely availability of high quality wireless connectivity the offering of which today is dominated by mobile network operators (MNOs). Future 5G systems aim at connecting billions of devices to serve versatile location and case specific needs of vertical sectors in parallel with the provisioning of traditional mobile broadband services. As the majority of mobile traffic originates from indoors, cost-efficient and fast deployment of new indoor small cell networks is fundamental, which calls for new developments in regulation and technology to enable new business. This paper proposes the concept of micro operators $(\mathrm{uO})$ for local service delivery in $5 \mathrm{G}$ to build indoor small cell communication infrastructure and offer context related services and content. Key elements of the new micro operator concept are introduced including regulation-related factors of local spectrum access rights, and technology-related factors of flexible network implementation. Several business opportunities are identified for the $\mathrm{uO}$ concept including the provisioning of hosted local connectivity to all MNOs in specific locations, operation of secure networks for vertical sector specific use, and offering of locally tailored content and services.
\end{abstract}

Keywords: 5G; business model; mobile communication network; mobile network operator; spectrum sharing;

Acknowledgments: Authors would like to acknowledge Tekes - the Finnish Funding Agency for Innovation in "Micro-operator concept for boosting local service delivery in 5G (uO5G)" project. 


\section{Introduction}

Increasing digitalization requires that versatile location and case specific requirements are met in different environments, such as hospitals, campuses, shopping malls, sports arenas, utilities, and factories with high traffic densities particularly in indoors. This places increasing requirements for wireless connectivity in terms of reliability, high quality, versatile data rates, low latency, security, and privacy up to a level that is beyond current wireless technology and mobile network operator (MNO) business models. The fifth generation (5G) mobile communication networks aim at accommodating a wide range of use cases with advanced requirements arising from various vertical sectors such as health, media, factories, and automotive [1]. 5G will provide end-to-end network and cloud infrastructure slices to fulfil vertical sector specific requirements and mobile broadband services in parallel [1].

Today's wireless mobile connectivity market is dominated by big MNOs whose business model is to offer services to the masses with high infrastructure investments and long investment cycles [2]. In the future, this model will become insufficient and various verticals and services cannot develop unless future wireless systems can rapidly respond to case-specific, increasing and versatile local traffic requirements.

Furthermore, currently the entry barrier to the mobile market is high due to the limited availability of spectrum licenses and the high infrastructure investments required. Spectrum licenses are limited in number, high-cost, long-term and often include nation-wide coverage obligations, which complicates the entry of new players to the market. As growing amount of mobile traffic originates from local indoor usage, serving the high traffic hot spots with traditional macro cellular networks becomes insufficient when the building penetration losses limit the indoor connectivity. To conclude, the construction, deployment and operation of indoor small cell networks should be made easy, cost-efficient and flexible to meet the facility owners and customers' - both organisations or individuals-needs and shift the focus from providing traditional mobile broadband services to meeting the local case-specific requirements of different vertical sectors. For efficient operation, this calls for changes in MNO business models and regulation towards rapid and localized service delivery and spectrum licensing.

Future mobile communication business ecosystem is expected to expand from static MNO dominance [2] to a universe of specialised companies providing services at different positions of the value chain [1] to complement the traditional mobile connectivity provisioning. The convergence of telecommunication, media and internet business domains into a sharing economy with rapid development cycles and regulatory initiatives on flexible sharing based local licensing are disrupting the traditional MNO dominant mobile connectivity business and open the market to new entrants. This disruption creates a new connectivity market where customers are the different industry verticals with highly distinct requirements for fast and scalable deployment of new digital services in local areas to speed up their adoption. In the changing business ecosystem around mobile connectivity, the role of facility owners and users becomes increasingly important in the development, operation and maintenance of indoor networks and offering of local content and services. Furthermore, the introduced change will extend the business model dynamics from connectivity towards Internet business models where additionally content, context and commerce layers are included resulting in ecosystemic $4 \mathrm{C}$ business model typology [3].

Understanding the changing mobile connectivity business ecosystem and operators' strategic choices in it requires a framework that consist of three inter-related domains: regulation, technology and business [2]. Regulation defines the boundaries for operations in terms of deciding who is allowed access the mobile market and under which conditions by e.g. granting spectrum licenses and defining rules for competition and collaboration. Technology domain includes the elements scope of innovation, maturity of technology, stage of standardization, and scale of use. The business perspective is related to logic and relationships of the players participating in value creation and capture, and is often characterized with business models and is strongly influenced by regulatory and technological developments. 
This paper analyses the changing mobile communication business ecosystem and identifies the emergence of a new role, namely the local micro operator $(\mathrm{uO})$, for addressing the vertical sector specific local connectivity needs continuing the work started in [4]. The paper depicts the underlying regulatory and technical developments towards $5 \mathrm{G}$ and identifies business opportunities for the newly proposed micro operators.

The rest of this paper is organized as follows. Section 2 introduces the new micro operator concept and its relations to other stakeholders. Section 3 describes the regulatory developments with a focus on spectrum regulation. Section 4 identifies technical developments enabling the micro operator concept. Section 5 identifies business opportunities based on the regulatory and technical developments. Finally, conclusions are drawn in Section 6.

\section{Micro operator concept}

This section depicts the changing mobile business ecosystem, introduces the new micro operator concept and positions it in the mobile business ecosystem.

\subsection{Changing mobile business ecosystem}

Traditional mobile communication business ecosystem [5-6] is at a turning point with the advent of 5G. Convergence of telecom and internet domains and changes in regulation are disrupting the traditional MNO dominant mobile connectivity business and opening the market to new entrants. The traditional stakeholder roles are changing and new roles are emerging. As the majority of traffic originates from indoors, the specifics of building indoor small cell networks must be considered. This disruption is creating a connectivity market where different vertical sectors have growing and versatile requirements for fast and scalable deployment of new digital services in local areas, which calls for renewed and complementary business models compared to MNOs' current business models.

Fig. 1 illustrates the changes in the mobile connectivity market. With the need to provide vertical specific locally tailored wireless connectivity services, the MNO market dominance with similar service offerings is seen to change into a local connectivity market where the customers are the different vertical sectors with versatile requirement for fast and scalable indoor small cell deployments. As the building of any new wireless network requires spectrum, the availability of local spectrum for small cell networks is a major challenge for the deployment of these new networks. Today's regulatory approach of auctioning a limited number of exclusive spectrum licenses to MNOs with long license durations and high coverage obligations that has led to the market dominance of a small number of MNOs is now changing towards opening the mobile connectivity market to new players with local spectrum access rights. Finally, the cost and complexity associated with all MNOs building separate indoor networks within the same facility can be circumvented by the virtualization and sharing economy approach where networks are scaled on-demand and assets currently owned by some players would be shared by others to create new revenue streams. This would lower the current entry barrier to the mobile market arising from the massive investments needed in the infrastructure, and reduce time to market.

Defining elements for the changing operator role in the future mobile business ecosystem include:

- Type or customer (machine, employee, another operator, customer of the operator, third party service provider, etc.)

- Specificity of requirements for the service (latency, security, service content, reliability, etc.)

- Need for and willingness to transparency of operations (openness, block chain).

- Co-opetition and partnering 
These changes will turn the mobile broadband business from competitive "value creation and capture" thinking towards co-opetitive value sharing economy and ecosystemic business models where a multitude of ecosystems collaborate and compete to complement the traditional mobile broadband provisioning.

TODAY

Big MNOs dominate the wireless connectivity

market and offer the

same services to masses.

Limited number of

spectrum licenses are

auctioned with long

license duration and high

coverage obligations.
FUTURE

New local connectivity

market is established where

verticals have versatile

requirements for fast and

scalable indoor deployment.

Changes in spectrum

regulation disrupt the

traditional mobile market by

opening it to new entrants.
Entry barrier to mobile market is high due to

high investment barrier

to build own

infrastructure.
Availability of local spectrum for small cell networks.
Provisioning of vertical connectivity services.


Sharing economy approach emerges where networks are scaled on demand and

assets currently owned by others are shared to make new revenues.

Fig. 1 Envisaged changes in the mobile connectivity market

\subsection{Micro operator key elements and relations}

This paper proposes the concept of micro-operators $(\mathrm{uO})$ to build indoor small cell communication infrastructure and offer local connectivity, context related services and content to complement existing mobile connectivity service offerings with the help of emerging $5 \mathrm{G}$ technology and locally issued spectrum licenses. We build our work upon the initial notion of micro operator introduced in [4] that defined the micro operator as an entity that combines connectivity with specific content services in spatially confined domains and is dependent on appropriate spectrum resources. The uO can establish local small cell networks that can serve the increasing customer demand with guaranteed quality through collaboration with network infrastructure vendors, facility users and owners, utility service companies, regulators, device and equipment vendors, content providers and MNOs.

Opposite to mobile virtual network operators (MVNOs), uOs have their own infrastructure to support any MNO customers for efficient local service delivery and complement the MNOs by building the infrastructure in places with case specific customer demands where traditional MNO business models for serving the masses become too rigid. They would benefit the major MNOs by offering them indoor capacity in high traffic demand areas. With the help of an agile local $\mathrm{uO}$, various facility owners and users could have an important role in building the required indoor small cell communication infrastructure as well as offering context related services and content in local areas to different customers in different vertical sectors. The increasing role of big data and analytics in telecommunications offers the uOs a unique opportunity to 
combine its telecommunication data about connectivity with the data collected from its customers into unique and tailored local service offerings in a certain location with guaranteed quality. The proposed uO concept will allow timely on-demand availability of capacity for local service delivery and rapid development cycles for future service provisioning for real digitalization across different verticals.

As a new role in the mobile connectivity market, the uO's relations to other key stakeholders are critical for the success of the concept. Examples of these relations are depicted in Fig. 2. Network infrastructure vendors provide scalable small cell networks and infrastructure for the $\mathrm{uO}$ on demand. Facility users and owners give the $\mathrm{uO}$ the permission to build the indoor small cell networks and obtain local services and content from the $\mathrm{uO}$. Regulator issues local spectrum licenses for the $\mathrm{uO}$ and defines the regulatory regime under which the $\mathrm{uO}$ must operate. If needed, spectrum brokers could manage spectrum resources on behalf of the regulator and spectrum users in order to allow dynamic management of the spectrum resources. Device and equipment vendors provide various devices that connect to the uO network to provide or use data. Content providers deliver locally tailored services and content to the $\mathrm{uO}$ to be offered to customers. Finally, the MNOs can buy capacity from the $\mathrm{uO}$ to serve their customers in specific areas and uOs can gain access to MNO infrastructure in other areas. Since the uO role is new, it is not yet tied to any specific stakeholder and can be taken by any of the existing or new stakeholders.

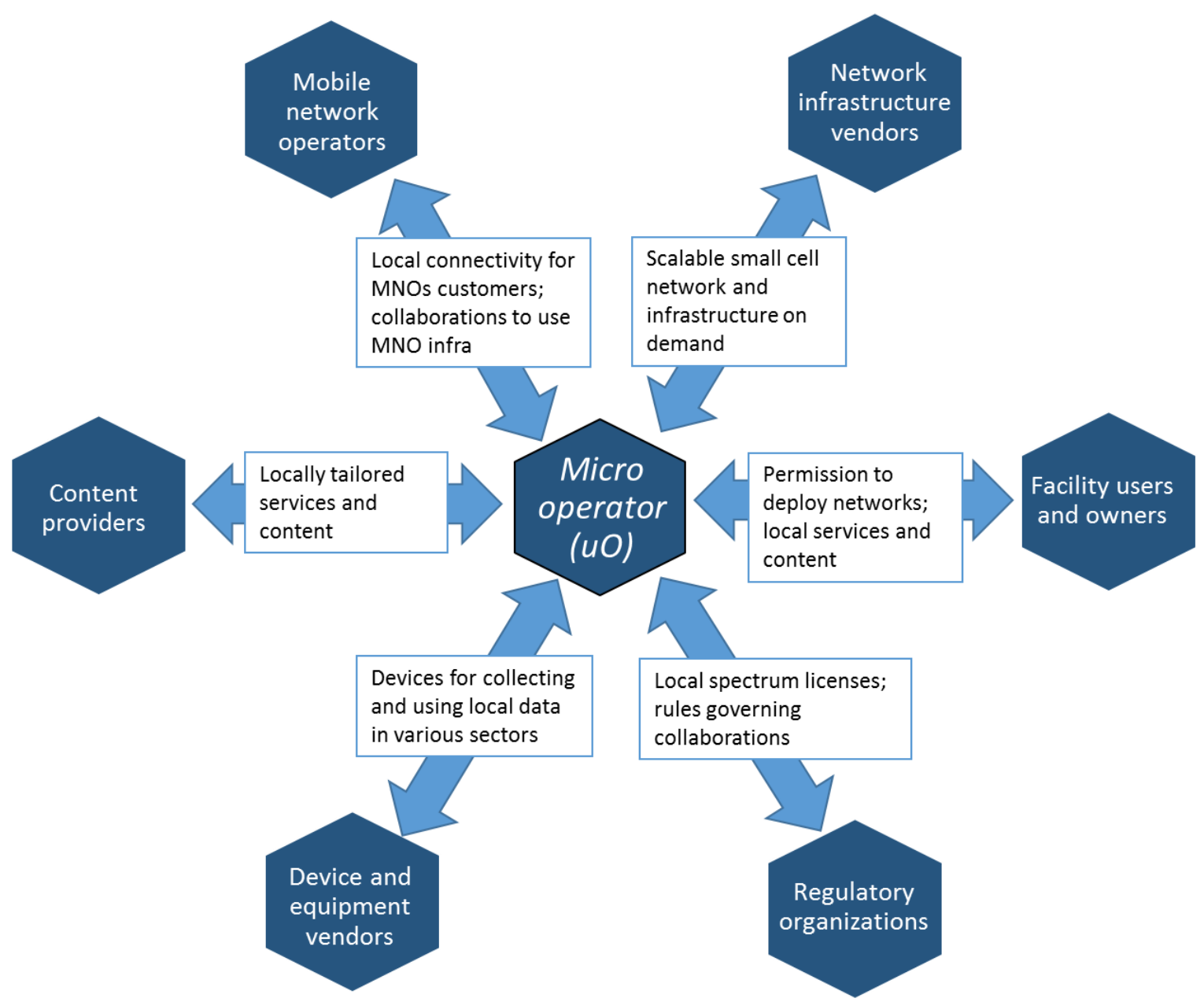

Fig. 2 Micro operator's relations with key stakeholders 


\section{Regulatory developments}

Spectrum regulatory developments have evolved from the traditional split between individual authorization (licensed) and general authorization (unlicensed) approaches towards light licensing [7] and spectrum sharing [8] where multiple systems operate in the same band under agreed rules and conditions. Traditionally, MNOs have obtained individual licenses which have recently been granted through auctions. Typically, due to the restricted carrier bandwidths available in a given frequency band the licenses are granted only to a small number of operators and often come with strict obligations on e.g. coverage. The same trend is continuing such as in the upcoming $700 \mathrm{MHz}$ band in Europe where typically there is room for feasible deployment by only three national operators since the band needs to be divided among them. Additionally, various stakeholders have set up wireless local area networks (WLAN) in the unlicensed bands to cover specific areas, such as hotels, enterprises, shopping malls, university campuses, and factories, but without quality of service (QoS) guarantees.

As the majority of traffic originates from indoors, development of regulation that improves the feasibility of building indoor connectivity solutions deserves increasing attention. Globally, 5G developments in regulation at the International Telecommunication Union Radiocommunication sector (ITU-R) are aiming at operations in higher frequency bands (millimetre wave spectrum bands) which are better suited for local and shared operation due to their propagation characteristics that restrict interference to local areas. World Radiocommunication Conference in 2019 (WRC-19) of the ITU-R will consider additional spectrum allocations to the mobile service on a primary basis and to consider identification of frequency bands for the terrestrial component of International Mobile Telecommunications (IMT) systems in the frequency range $24-86 \mathrm{GHz}$. The bandwidths available in these higher frequency bands are significantly wider compared to existing spectrum bands used for mobile communications. An open issue is the licensing of these bands as the traditional conditions with nation-wide coverage obligations do not apply and unlicensed access alone does not fulfil all the upcoming $5 \mathrm{G}$ requirements. To this end, the Federal Communications Commission (FCC) in the US has announced to open up a total of $10.85 \mathrm{GHz}$ of $5 \mathrm{G}$ spectrum in the frequency range between 27.5 and $81 \mathrm{GHz}$ including $3.85 \mathrm{GHz}$ of licensed flexible use spectrum and $7 \mathrm{GHz}$ of unlicensed spectrum [9]. This growing interest towards higher frequency bands is increasing the feasibility of building indoor small cell networks.

Wider bandwidths are also available in the frequency bands between existing mobile communication bands and the future millimetre wave bands, such as the $3.4-4.2 \mathrm{GHz}$ frequency band whose parts are under active study in Europe [10] and US [11-12]. In many countries there are incumbent systems whose rights as current license holders need to be preserved. To protect the incumbents, spectrum sharing models have been developed to speed up the process of gaining access to bands that currently are underutilized by incumbents. These models include Licensed Shared Access (LSA) in Europe [13-15] and three-tier Citizens Broadband Radio Service (CBRS) sharing framework in the US [11-12] whose regulation and standardization have progressed in $2.3-2.4 \mathrm{GHz}$ and $3.6-3.8 \mathrm{GHz}$ bands in Europe and in 3.55-3.70 GHz band in US, respectively. The LSA sharing model introduces additional licensed users in bands in accordance with agreed sharing rules that allow both incumbent and additional users to provide a certain QoS [13]. The US three-tier sharing framework introduces flexible licensing models to open the market to new entrants including a layer of local priority access licenses (PAL) and a layer of general authorized access (GAA) usage coordinated by the Spectrum Access Systems (SAS).

With the above spectrum regulatory developments in mind, the emergence of the new uO concept introduced in Chapter 2 is dependent on the regulation through the availability of local micro licenses and the availability of feasible regulation for building and operations of indoor small cell networks. The availability of local micro licenses is a new paradigm shift that is occurring in the global scale as the existing exclusive licensing models with coverage obligations will result in a mismatch with the operational 
characteristics of higher frequency bands. The recent regulatory developments on spectrum sharing including LSA in Europe and three-tier sharing framework in the US introduce flexible licensing models to open the market to new entrants particularly around the $3.5 \mathrm{GHz}$ band. This is a potential band for the trialing of the $\mathrm{uO}$ operations as the $3.5 \mathrm{GHz}$ band is actively used for $5 \mathrm{G}$ test networks in Finland. If incumbent protection is needed, the LSA model could be used to allow local uO operations while protecting the active incumbents and studied for 3.6-3.8 GHz band in Europe [10].

In addition to spectrum, there is other regulation that touches the building and operation of indoor small cell networks. The regulations to govern the construction and deployment of indoor networks vary from one country to another and the availability of suitable spectrum for them has so far mainly limited to unlicensed bands which has restricted the current deployments. Several regulation-related topics need to be considered such as national roaming for connecting the users to mobile communication networks outside the uOs coverage area, rights to build indoor small cell networks and facility owners' role in it, conditions for using mass data collected from users, and net neutrality among others. Moreover, regulation governing the new sharing economy is open and creation of new rules for assigning local spectrum access rights through flexible licensing is topical for $5 \mathrm{G}$ bands.

\section{Technology developments}

The next big technical leap in mobile communication developments comes with $5 \mathrm{G}$ that aims at connecting billions of devices anytime anywhere to meet vertical sectors' requirements in addition to the traditional mobile broadband services [1]. $5 \mathrm{G}$ technology developments include two parallel threads: the evolution of fourth generation (4G) long-term evolution (LTE) based networks and a new air interface to operate in the higher frequency bands $(24-86 \mathrm{GHz})$. $5 \mathrm{G}$ will become a part of heterogeneous networks to connect a vast amount of devices to the Internet via distributed and flexible allocation of network functions [1]. Technical developments in 5G will introduce network function virtualization (NFV) and slicing functionalities that will allow the leasing of the required parts of the communications infrastructure without extensive investments in the infrastructure. 5G architecture will integrate dense small cell networks in higher frequency bands with MNOs' existing heterogeneous networks that consist of multiple overlapping cell layers in various frequency bands. It will accommodate a wide range of use cases with advanced requirements, especially in terms of latency, data rates, user density, mobility, and coverage [16].

Future $5 \mathrm{G}$ networks are expected to operate in a wide range of frequency bands including bands below 1 $\mathrm{GHz}$, between 1-6 GHz and above $6 \mathrm{GHz}$. New spectrum bands envisaged for $5 \mathrm{G}$ are sought in the frequency range between $24 \mathrm{GHz}$ and $86 \mathrm{GHz}$ which were not used for cellular systems before, and there is a need to develop the required technology to operate mobile communication systems in these bands. New innovations are particularly envisaged for this part of $5 \mathrm{G}$ development including e.g. new architecture, mobile edge computing, network slicing, software defined networking, massive MIMO, beam forming and wireless backhauling [17-21]. Wider carrier bandwidths and more efficient frequency reuse are possible in these higher frequency bands which facilitate local licensing models.

An important technical development is network slicing which is a collection of mobile network functions and a specific set of radio access technologies (RATs) necessary to operate an end-to-end logical mobile network [21]. This network slice can be tailored to the requirements of considerably different use cases, network customers, or business models. NFV would allow the local operators to lease the required network slices for provisioning of their services and content. In addition the dense small cell indoor networks require efficient backhauling which could be wireless that would ease the installations of a large number of small cells, see e.g. [18-19]. 
Spectrum sharing techniques developed for LSA and the 3-tier sharing framework will be available in 5G networks to protect incumbents and adjust the networks according to changing spectrum availability and interference conditions by turning on/off selected base stations, lower transmission powers, etc. Database based approaches for managing access to shared spectrum in LSA and three-tier model are developed to be supported by the cellular architecture evolutions.

To conclude, the global $5 \mathrm{G}$ developments including network slicing and virtualized network functions, mobile edge computing capabilities, and higher carrier frequencies facilitate the emergence of the new uO role for locally operated small cell networks. The new $5 \mathrm{G}$ mobile network functions can offer the $\mathrm{uO}$ a platform to take full advantage of the localized shared spectrum and telecommunication cloud resources with rapid content processing delivering ultra-responsive experience. The $\mathrm{uO}$ concept will benefit from the $5 \mathrm{G}$ technical developments by means of being able to lease the required parts of the infrastructure locally on demand without extensive investments in infrastructure and long-term spectrum licenses.

\section{Business opportunities}

This section analyses the business opportunities arising from the on-going regulatory and technology developments depicted in Sections 3 and 4 for the new micro operator concept introduced in Section 2 . In terms of business opportunities, $5 \mathrm{G}$ promises universal availability of instantaneous communications, high level of guaranteed QoS, and cost levels appropriate for meeting customers' expectations [1]. 5G aims at opening up new business opportunities by providing end-to-end network slices to fulfil vertical specific requirements and mobile broadband services [1]. In parallel with provisioning of mobile broadens services, $5 \mathrm{G}$ will serve the versatile requirements arising from different verticals. The sharing economy approach is entering the mobile business, and R\&D efforts on $5 \mathrm{G}$ are offering new functions that allow lower investments to access the mobile market. This results in new business relations between stakeholders in the mobile business ecosystem.

As growing amount of mobile traffic originates from indoors and cost-efficient provisioning of indoor capacity is needed in different industry verticals for digitalization, the timely provisioning of cost-efficient wireless connectivity becomes increasingly interesting business opportunity. While the MNO business continues to stay strong, there are new business opportunities for agile local players to offer local connectivity and case-specific tailored services in rapid time scales to speed up the adoption of digitized services.

The proposed $\mathrm{uO}$ concept is expandable to a variety location and context specific use cases in e.g. hospitals, campuses, shopping malls, and mass event arenas, to respond to the distinct business related requirements of e.g. automotive, media, entertainment, health, utility, and factory vertical sectors, see e.g. [22-23]. Detailed understanding of the case specific services or processes to be controlled is vital for making efficient verticals reality. In many cases MNOs do not have the required understanding or the market is simply too small for them to get interested as their business models are based on high infrastructure investments to serve the masses.

The following four business opportunities can be identified for uOs:

1) Offering hosted local connectivity to all MNOs in specific locations. uO can operate local small cell networks in selected facilities to serve other MNOs' customers. Construction of indoor small cell networks is not cost-efficient and feasible if all MNOs build their separate indoor networks. MNOs can encounter significant cost savings by letting the local connectivity be handled by uOs in collaboration with facility owners and buy the required capacity from the uO. 
2) Secure local networks for vertical specific needs. uO can operate local small cell networks in specific facilities offering a secure closed local network to cover the vertical sector specific service chain with predefined access to the outside world in e.g. factories, and hospitals.

3) Locally tailored services. uO can offer locally tailored services through its network in local areas, e.g., premium content services or relevant context specific information. Delivering services, such as extremely high data volume, throughput and consistent quality of experience ( $\mathrm{O} O E)$ broadband, ultra-high definition (UHD) video and virtual/augmented reality (VR/AR), in a managed way can open up new monetization opportunities in partnership with content providers and global content delivery networks (CDN) by providing a clear value add to the partners in the value chain and ultimately the end users.

4) MyData operator. uO can act as a "my data operator" and govern the data of certain applications and individual users while at the same time offering connectivity in the local area. MyData is a novel approach to human centered personal data management. In a long run MyData will enable wider personal data availability and hence provide new types of business opportunities. Recent study [24] showed there to be two types of general-level business models for MyData platform operators: service-based and transactionbased. In a service-based business model, the platform operators generate revenue by offering valueadding services on top of free services for platform users (both individual users and companies). In transaction-based business model revenue is generated through fees from facilitating data transactions and operating the platform. The transaction-based business model is based on data brokering service, which can leverage transactional and control data produced by the stakeholders to enable entirely new services that benefit from contextual real-time and non real-time data. This could enable 'Platform as a service' kind of business models, where operators can securely expose service and network assets to third parties, such as loT platforms or location based applications. Complex big data analytics and machine learning can be applied to anonymize and aggregate network signaling data for deriving insights useful for third party services or enterprises. Operators can broker transactional and control data produced by the network to different industries: providers of augmented reality services, traffic steering systems (e.g. provided by municipalities), factories and logistical systems and utilities.

By using the $4 \mathrm{C}$ business model logic [3] consisting of connectivity, content, context and commerce layers, the $\mathrm{uO}$ can offer services in all of the four layers as initially depicted in [4]. The above described uO business opportunities can be grouped as presented in Fig. 3 . 


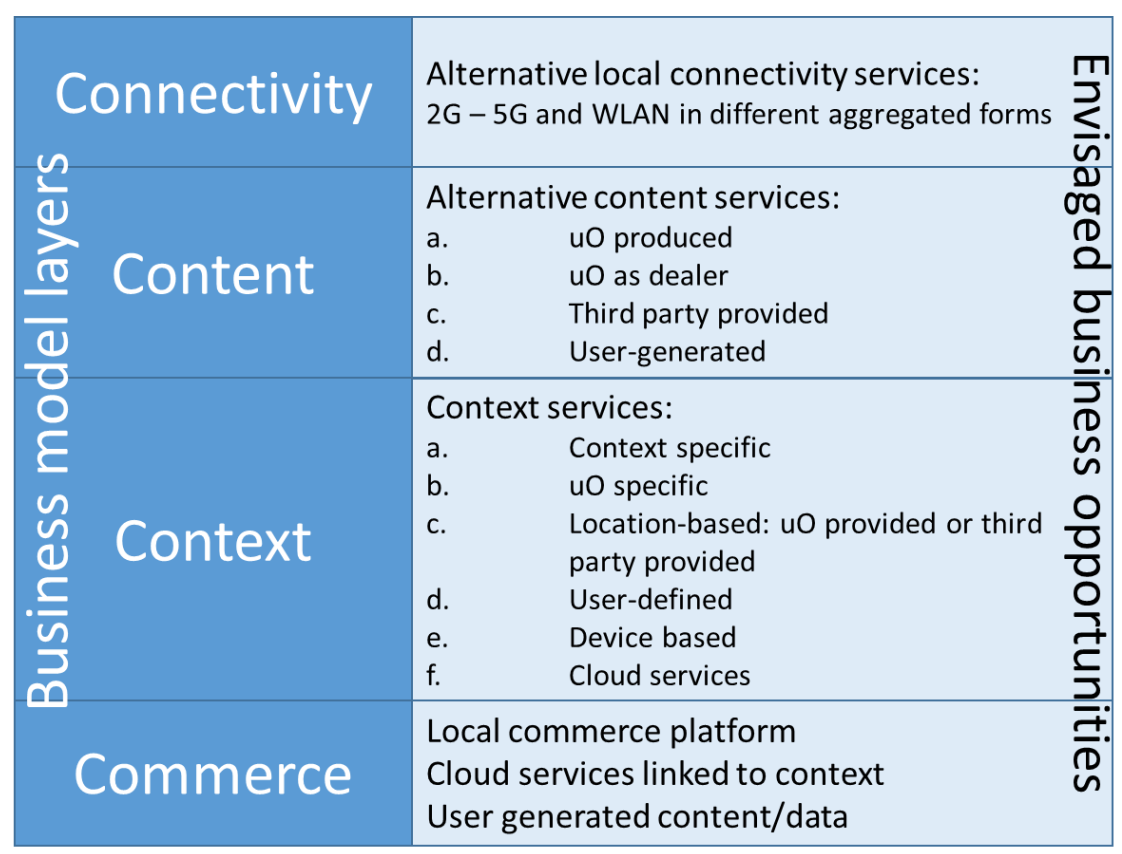

Fig. 3 uO business opportunities

To conclude, several business opportunities are seen to arise for the uOs ranging from serving other MNOs customers to providing closed vertical specific local networks. The uOs can have different offerings in terms of connectivity, content, context and commerce services. In all the different service offerings, a key aspect becomes the relation of the $\mathrm{uO}$ with the other key stakeholders in terms of what the $\mathrm{uO}$ can offer for the stakeholders and what it requires from them. This will result in co-opetitive relations [25] between stakeholders where both co-operative and competitive relationships simultaneously exist.

\section{Conclusions}

This paper has introduced the new micro operator $(\mathrm{uO})$ concept for local service delivery in future $5 \mathrm{G}$ mobile communication business ecosystem. The developed micro operator concept has its basis in the ongoing regulatory and technical developments towards local spectrum access rights and flexible network implementations to open the mobile communication market to business opportunities that could be seized by the new micro operators. These business opportunities arise from applying mobile connectivity to the various vertical sectors' needs, which calls for local agile players with scalable business models extending from connectivity to content, context and commerce layers. The first concrete target for trialing the micro operator concept is showcasing the feasibility of local operations in the $3.5 \mathrm{GHz}$ band using $5 \mathrm{G}$ test networks with locally issued spectrum licenses. Future work is needed to develop contents for the scalable business models that can be timely adapted to the vertical specific conditions and requirements and trial the feasibility of the concept in practise.

\section{References}

1. 5GPPP. (2016). 5G empowering vertical industries. Roadmap paper. The $5 \mathrm{G}$ Infrastructure Public Private Partnership. https://5g-ppp.eu/wpcontent/uploads/2016/02/BROCHURE 5PPP BAT2 PL.pdf Accessed 15 July 2016. 
2. Ahokangas, P., Matinmikko, M., Yrjölä, S., Okkonen, H., \& Casey, T. (2013). "Simple rules" for mobile network operators' strategic choices in future cognitive spectrum sharing networks. IEEE Wireless Communications, 20(2), 20-26.

3. Wirtz, B. W., Schilke, O., \& Ullrich, S. (2010). Strategic development of business models: Implications of the Web 2.0 for creating value on the internet. Long range planning, 43(2), 272290.

4. Ahokangas, P., Moqaddamerad, S., Matinmikko, M., Abouzeid, A., Atkova, I. Francis Gomes, J., \& livari, M. (2016). Future micro operators business models in 5G. In International conference on Restructuring of the Global Economy (ROGE).

5. Basole, R. C. (2009). Visualization of interfirm relations in a converging mobile ecosystem. Journal of Information Technology, 24(2), 144-159.

6. Al-Debei M.M., Al-Lozi E., \& Fitzgerald, G. (2013). Engineering innovative mobile data services. Business Process Management Journal, 19(2), 336-363.

7. CEPT ECC. (2009). Light licensing, license-exempt and commons. ECC Report 132. European Conference of Postal and Telecommunications, Electronic Communications Committee.

8. ITU-R. (2014). Cognitive radio systems in the land mobile service. Report ITU-R M.2330. International Telecommunication Union Radiocommunication sector.

9. FCC. (2016). Fact sheet: Spectrum frontiers rules identify, open up vast amounts of new high-band spectrum for next generation (5G) wireless broadband. Federal Communications Commission. http://transition.fcc.gov/Daily Releases/Daily Business/2016/db0714/DOC-340310A1.pdf Accessed 15 July 2016.

10. CEPT ECC. (2016). Operational guidelines for spectrum sharing to support the implementation of the current ECC framework in the $3600-3800 \mathrm{MHz}$ range. ECC Report 254. European Conference of Postal and Telecommunications, Electronic Communications Committee.

11. FCC. (2015). Amendment of the Commission's Rules with Regard to Commercial Operations in the 3550-3650 MHz Band, Report and Order and Second Further Notice of Proposed Rulemaking. FCC15-47. Federal Communications Commission.

12. FCC. (2016). Amendment of the Commission's Rules with Regard to Commercial Operations in the 3550-3650 MHz Band, Order of Reconsiderations and Second Report and Order. FCC-16-55. Federal Communications Commission.

13. RSPG. (2013). RSPG Opinion on Licensed Shared Access. RSPG13-538. European Commission, Radio Spectrum Policy Group.

14. CEPT ECC. (2014). Licensed shared access. ECC Report 205. European Conference of Postal and Telecommunications, Electronic Communications Committee.

15. ETSI. (2015). System Architecture and High Level Procedures for operation of Licensed Shared Access (LSA) in the $2300 \mathrm{MHz}-2400 \mathrm{MHz}$ band. ETSI TS 103 235. European Telecommunications Standards Institute.

16. ITU-R. (2015). IMT Vision - Framework and Overall Objectives of the Future Development of IMT for 2020 and Beyond. Recommendation ITU-R M.2083-0. International Telecommunication Union Radiocommunication sector.

17. Wang, C.-X., Haider, F., Gao, X., You, X.-H., Yang, Y., Yuan, D., Aggoune, H. M., Haas, H., Fletcher, S., \& Hepsaydir, E. (2014). Cellular architecture and key technologies for $5 \mathrm{G}$ wireless communication networks. IEEE Communications Magazine, 52(2), 122-130.

18. Dehos, C., González, J. L., De Domenico, A., Kténas, D., \& Dussopt, L. (2014). Millimeter-wave access and backhauling: the solution to the exponential data traffic increase in $5 \mathrm{G}$ mobile communications systems? IEEE Communications Magazine, 52(9), 88-95.

19. Ge, X., Cheng, H., Guizani, M., \& Han, T. (2014). 5 G wireless backhaul networks: Challenges and research advances. IEEE Network, 28(6), 6-11.

20. Nguyen, V.-G., Do, T.-X, \& Kim, Y. (2016). SDN and virtualization-based LTE mobile network architectures: A comprehensive survey. Wireless Personal Communications, 86(3), 1401-1438. 
21. Rost, P., Berberana, I., Maeder, A., Paul, H., Suryaprakash, V., Valenti, M., Wübben, D., Dekorsy, A., \& Fettweis, G. (2015). Benefits and challenges of virtualization in $5 \mathrm{G}$ radio access networks. IEEE Communications Magazine, 53(12), 75-82.

22. 5GPPP. (2015). 5G and eHealth. White paper. The $5 \mathrm{G}$ Infrastructure Public Private Partnership. https://5g-ppp.eu/wp-content/uploads/2016/02/5G-PPP-White-Paper-on-eHealth-VerticalSector.pdf Accessed 15 July 2016.

23. 5GPPP. (2016). 5G and Media \& Entertainment. White paper. The 5G Infrastructure Public Private Partnership. https://5g-ppp.eu/wp-content/uploads/2016/02/5G-PPP-White-Paper-on-MediaEntertainment-Vertical-Sector.pdf Accessed 15 July 2016.

24. Kemppainen, L., Pikkarainen, M. \& Koivumäki, T. (2016). Business models for platform operators in the field of human-centered personal data management: A Case Study Approach. Research publication, Digital Health Revolution project, University of Oulu.

25. Gnyawali, D. \& Park, B. (2009). Coopetition and technological innovation in small and medium-sized enterprises: A multilevel conceptual model. Journal of Small Business Management, 47(3): 308330. 